\title{
Avaliação da implantação do acesso avançado nos indicadores de qualidade de uma unidade de saúde
}

\author{
Evaluation of the implementation of advanced access on the quality indicators of a health unit
}

\section{Evaluación de la implementación del acceso avanzado en los indicadores de calidad de una unidad de salud}

Pedro Pablo de Gusmão Bonilla'® ${ }^{1}$, Lucas Wollmann

${ }^{1}$ Grupo Hospitalar Conceição

\section{Resumo}

Objetivos: O objetivo principal foi analisar os indicadores de acesso da unidade de saúde (US) Sesc, antes e após a implantação do acesso avançado (AA). Como objetivo secundário, foram analisados os indicadores de desempenho da US Sesc, antes e após o AA. Métodos: Estudo transversal descritivo com análise dos dados obtidos mensalmente do sistema de informação em saúde do Grupo Hospitalar Conceição, comparando o período de novembro/16 -outubro/17 com novembro/17 - outubro/18. Resultados: Após o AA, o número de atendimentos médicos aumentou $8 \%$, o absenteísmo nas consultas diminuiu de $6,9 \%$ para $3 \%$ e o número de pacientes diferentes atendidos teve um acréscimo de quase $5 \%$. Em relação ao controle de doenças crônicas, o número de hipertensos atendidos cresceu e também houve um aumento na porcentagem de hipertensos controlados, passando de $74 \%$ para $77 \%$. O número de diabéticos atendidos aumentou, assim como a porcentagem de diabéticos controlados subiu de $73 \%$ para $81 \%$. Houve uma diminuição de quase $10 \%$ de atendimentos a pacientes vinculados à US nos serviços de atenção secundária de referência. Discussão: É possível considerar que a implantação do AA na US Sesc foi associada a uma melhoria dos indicadores de acesso, bem como da maior parte dos indicadores de desempenho analisados.

Palavras-chave: Acesso Avançado; Indicadores e Reagentes; Centros de Saúde.

\section{Abstract}

Objectives: The main objective was to analyze the access indicators of the Sesc health unit (HU), before and after the implementation of advanced access (AA). As a secondary objective, the performance indicators of the health unit Sesc HU were analyzed, before and after AA. Methods: Cross-sectional descriptive study with analysis of data obtained monthly from the health information system of Grupo Hospitalar Conceição, comparing the period of November/16 - October/17 with November/17 - October/18. Results: After AA, the number of medical appointments increased by $8 \%$, absenteeism in consultations decreased from $6.9 \%$ to $3 \%$ and the number of different patients treated increased by almost $5 \%$. Regarding the control of chronic diseases, the number of hypertensive patients seen increased and there was also an increase in the percentage of controlled hypertensive patients, from $74 \%$ to $77 \%$. The number of diabetics treated increased, as did the percentage of controlled diabetics increased from $73 \%$ to $81 \%$. There was a decrease of almost $10 \%$ in attendance to patients linked to the US in the reference secondary care services. Discussion: It is possible to consider that the implantation of AA in Sesc HU was associated with an improvement in access indicators, as well as most of the performance indicators analyzed.

Keywords: Advanced Access; Indicators and Reagents; Health Centers.

Como citar: Bonilla PPG, Wollmann L. Avaliação da implantação do acesso avançado nos indicadores de qualidade de uma unidade de saúde. Rev Bras Med Fam Comunidade. 2020;15(42):2360. https://doi.org/10.5712/rbmfc15(42)2360

Autor correspondente:

Pedro Pablo de Gusmão Bonilla.

E-mail: bonilla.pp@gmail.com

Fonte de financiamento:

declaram não haver.

Parecer CEP:

não se aplica.

Procedência:

não encomendado.

Avaliação por pares:

externa.

Recebido em: : 26/01/2020.

Aprovado em: 27/05/2020. 


\section{Resumen}

Objetivos: El objetivo principal era analizar los indicadores de acceso de la unidad de salud (US) Sesc, antes y después de la implementación del acceso avanzado (AA). Como objetivo secundario, se analizaron los indicadores de rendimiento de la US Sesc, antes y después del AA Método: Estudio descriptivo transversal con análisis de datos obtenidos mensualmente del sistema de información de salud del Grupo Hospitalar Conceição, comparando el período Noviembre/16 - Octubre/17 con Noviembre/17 - Octubre/18. Resultados: Después del AA, el número de citas médicas aumentó en un $8 \%$, el absentismo en las consultas disminuyó del 6,9\% al 3\% y el número de pacientes diferentes tratados aumentó en casi $5 \%$. Con respecto al control de enfermedades crónicas, el número de pacientes hipertensos vistos aumentó y también hubo un aumento en el porcentaje de pacientes hipertensos controlados, del $74 \%$ al $77 \%$. El número de diabéticos tratados aumentó, al igual que el porcentaje de diabéticos controlados aumentó del $73 \%$ al $81 \%$. Hubo una disminución de casi $10 \%$ en la asistencia a pacientes vinculados a la US en los servicios de atención secundaria de referencia. Discusión: Es posible considerar que la implantación del AA en la US Sesc se asoció con una mejora en los indicadores de acceso, así como en la mayoría de los indicadores de desempeño analizados.

Palabras clave: Acceso Avanzado; Indicadores y Reactivos; Centros de Salud.

\section{INTRODUÇÃO}

A atenção primária foi descrita na literatura internacional, a partir de seus atributos essenciais: o primeiro contato, a longitudinalidade, a integralidade e a coordenação do cuidado. O primeiro contato aborda a acessibilidade e o uso de serviços devido aos problemas para os quais se procura atenção à saúde. Ainda que o acesso não seja diretamente um dos atributos da atenção primária, ele está fortemente vinculado ao atributo essencial do primeiro contato, que consiste na ideia de que existe um ponto de entrada cada vez que um novo atendimento é necessário e que este ponto de entrada deve ser de acesso fácil à sua população adscrita. ${ }^{1}$

Para entender o acesso avançado, é útil primeiro entender as duas abordagens alternativas existentes para a gestão da demanda: o modelo tradicional e o modelo "carve-out" ou sistema com vagas.

A lógica do sistema tradicional é "faça o trabalho dos últimos meses, hoje". Esse sistema assenta-se no falso pressuposto da imprevisibilidade da demanda. Nele, todo dia a agenda está saturada por pessoas que foram marcadas previamente e pressionada, adicionalmente, pelos eventos agudos que surgem de forma não programada. Operacionalmente, o sistema tradicional divide as demandas em dois grupos: as demandas por condições urgentes que devem ser atendidas no mesmo dia e as demandas não urgentes que podem ser atendidas em outros dias. Para controlar a demanda, criam-se restrições no acesso e tipos de agendamento como os horários dos programas (gestantes, diabéticos, hipertensos, puericultura, etc). A forma de superar o desajuste entre demanda e oferta é por meio da redução da oferta. Esse sistema apresenta muitos problemas, pois ele gera excesso de atendimentos de eventos agudos que transgride o atributo essencial da longitudinalidade, reforça o comportamento da "tirania do urgente" e leva a altas taxas de absenteísmo, devido ao longo tempo para marcação das consultas. . $^{2,3,4}$

O sistema "carve-out" ou sistema com vagas, foi desenvolvido como alternativa ao sistema tradicional a partir dos estudos que demonstraram que a demanda na atenção primária à saúde é previsível. A lógica desse sistema é: "faça alguns trabalhos de hoje, hoje", ele representa uma melhoria em relação ao sistema tradicional, mas ainda apresenta alguns problemas. Pessoas com demandas não urgentes são agendadas para o futuro e o trabalho se acumula; esse sistema cria uma situação em que pessoas não podem ser atendidas no mesmo dia, mas não podem esperar o fim da fila, levando ao aumento dos tempos de espera e criando uma tensão entre os atendimentos de urgência e de não urgência. O pessoal do administrativo tem a tendência a sugerir que as pessoas telefonem ou apareçam no dia que querem ser atendidas, o que impacta na previsibilidade da demanda e cria problemas de agendamento no futuro. 
Assim, esse sistema reforça o comportamento clínico da "tirania do urgente", as pessoas definidas como portadoras de condições não urgentes, por serem incluídas em atendimentos futuros com tempos de espera longos, são induzidas a procurar as unidades de pronto atendimento ou os centros de atenção ambulatorial especializada que não são, em geral, adequados para uma resposta racional às suas demandas, o que acaba causando iatrogenia aos pacientes e maiores custos para o sistema de saúde..$^{3,4,5}$

Já o acesso avançado (AA) é um modelo de acesso que foi proposto nos Estados Unidos, no final da década de 90, disseminou-se para outros países como a Inglaterra e Austrália, e atualmente está presente em diversas unidades de saúde do Brasil. Esse modelo tem como premissa: "Faça hoje, o trabalho de hoje", permitindo que o usuário seja atendido pelo seu médico no mesmo dia ou em até 2 dias em que solicita o atendimento de qualquer natureza: eventos agudos, condições crônicas não agudizadas, cuidados preventivos e outros. Isso reduz o tempo de espera e aumenta a satisfação da equipe de atenção primária à saúde e das pessoas usuárias. ${ }^{6}$ Assim, o acesso avançado objetiva balancear as relações entre demanda e oferta, reduzir o intervalo de tempo entre a expressão da demanda e seu efetivo atendimento, reduzir ou estabilizar a demanda, aumentar a capacidade de oferta e possibilitar que as pessoas sejam atendidas pelos médicos aos quais estão vinculadas no mesmo dia, independentemente da natureza da demanda. ${ }^{7}$

A unidade de saúde (US) Sesc localiza-se na zona norte de Porto Alegre e possui uma população adscrita de cerca de 5.500 pessoas. Durante o período analisado, a US Sesc esteve aberta para atendimentos das $8 \mathrm{~h}$ às $18 \mathrm{hrs}$, de segunda a sexta-feira e possuía quatro médicos contratados, dois atendendo em cada turno, três enfermeiras contratadas, com pelo menos uma atendendo por turno, além de duas médicas residentes. Até o dia $1^{\circ}$ de novembro de 2017 , adotava o modelo "carve-out", com 50\% das consultas reservadas para demandas urgentes, nas quais o paciente era atendido no próprio dia e $50 \%$ para consultas para demandas não-urgentes, que eram agendadas no início de cada mês. Antes da implantação do acesso avançado foi feito um levantamento do número total de usuários cadastrados e o número de pacientes que tinham sido atendidos nos últimos 18 meses na unidade (usuários ativos), calculou-se a provável demanda de consultas por dia e concluiu-se que a equipe médica e de enfermagem teria condições de ofertar o número necessário de consultas para suprir a demanda. Após a decisão de implantar o acesso avançado, foram necessários mais 2 meses de preparação e planejamento com atividades semanais com a equipe de saúde (agentes comunitárias de saúde, técnicos de enfermagem, médicos, enfermeiras, auxiliares administrativos e residentes de medicina) para definição de vinculação dos usuários com suas equipes de referência, organização da agenda médica e de enfermagem, definição das atribuições do administrativo, acolhimento, enfermagem e médicos e, por fim, a pactuação com Conselho Local de Saúde (CLS).

O objetivo principal é analisar os indicadores de acesso da US Sesc, antes e após a implantação do AA. Como objetivo secundário, foram analisados os indicadores de desempenho da US Sesc antes e após a implantação do AA.

\section{MÉTODOS}

Este é um estudo transversal descritivo com análise dos dados obtidos mensalmente do Sistema de Informação em Saúde (SIS) do Serviço de Saúde Comunitária - Grupo Hospitalar Conceição (SSC-GHC) comparando o período de novembro/16 - outubro/17 com novembro/17 outubro/18. 
Os indicadores de acesso analisados foram: número de consultas feitas pela equipe médica e de enfermagem, número de pessoas diferentes atendidas e absenteísmo nas consultas. Os indicadores de desempenho analisados foram: número de exames citopatológicos (CPs) coletados em mulheres entre 25-64 anos, número de consultas de pacientes portadores de doenças crônicas (hipertensão, diabetes e asma), proporção de pacientes com hipertensão controlada ( $\mathrm{PA}<140 / 90 \mathrm{mmHg}$ ) e com diabetes controlado $(\mathrm{HbA} 1 \mathrm{c}<8,0 \%)$ e, além disso, foram pesquisados, através do SIS-GHC, o número de atendimentos e internações por condições sensíveis à atenção primária nos serviços de referência da US Sesc (Unidade de Pronto Atendimento da Zona Norte, Hospital Nossa Senhora da Conceição, Hospital da Criança Conceição, Hospital Cristo Redentor e Hospital Fêmina).

Este trabalho foi aprovado pelo Comitê de Ética e Pesquisa do GHC (parecer $n^{\circ}$ 3.281.396), não possuiu financiamento externo e os autores declaram não ter nenhum conflito de interesse.

\section{RESULTADOS}

Durante o período analisado, o número total de atendimentos médicos aumentou $8 \%$, enquanto os de enfermagem cresceram $61 \%$. O absenteísmo nas consultas médicas diminuiu de 6,9\% para $3 \%$, no entanto, quanto às consultas de enfermagem, houve um pequeno aumento de $3,7 \%$ para $4,1 \%$. O número de pacientes diferentes atendidos teve um acréscimo de quase $5 \%$ (Gráfico 1).

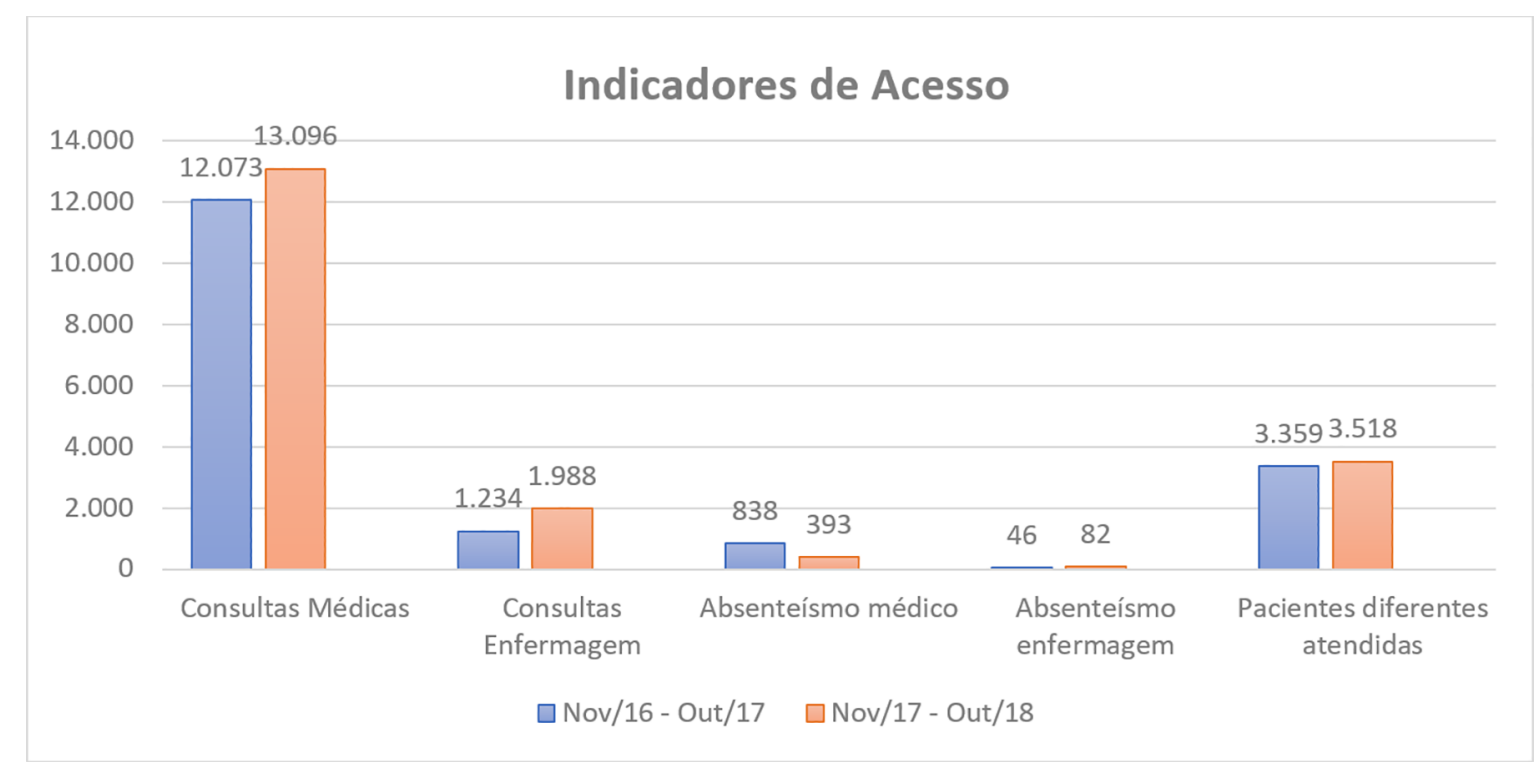

Gráfico 1. Indicadores de acesso antes e após a implantação do AA.

Em relação aos programas de controle de doenças crônicas, o número de hipertensos atendidos cresceu, assim como também houve um aumento na porcentagem de hipertensos controlados, passando de $74 \%$ para $77 \%$. O número de diabéticos atendidos aumentou, assim como a porcentagem de diabéticos controlados subiu de $73 \%$ para $81 \%$. A quantidade de crianças asmáticas em acompanhamento regular se manteve praticamente igual. Houve um decréscimo apenas no número de coleta de CPs em mulheres entre 25-64 anos (Gráfico 2). 


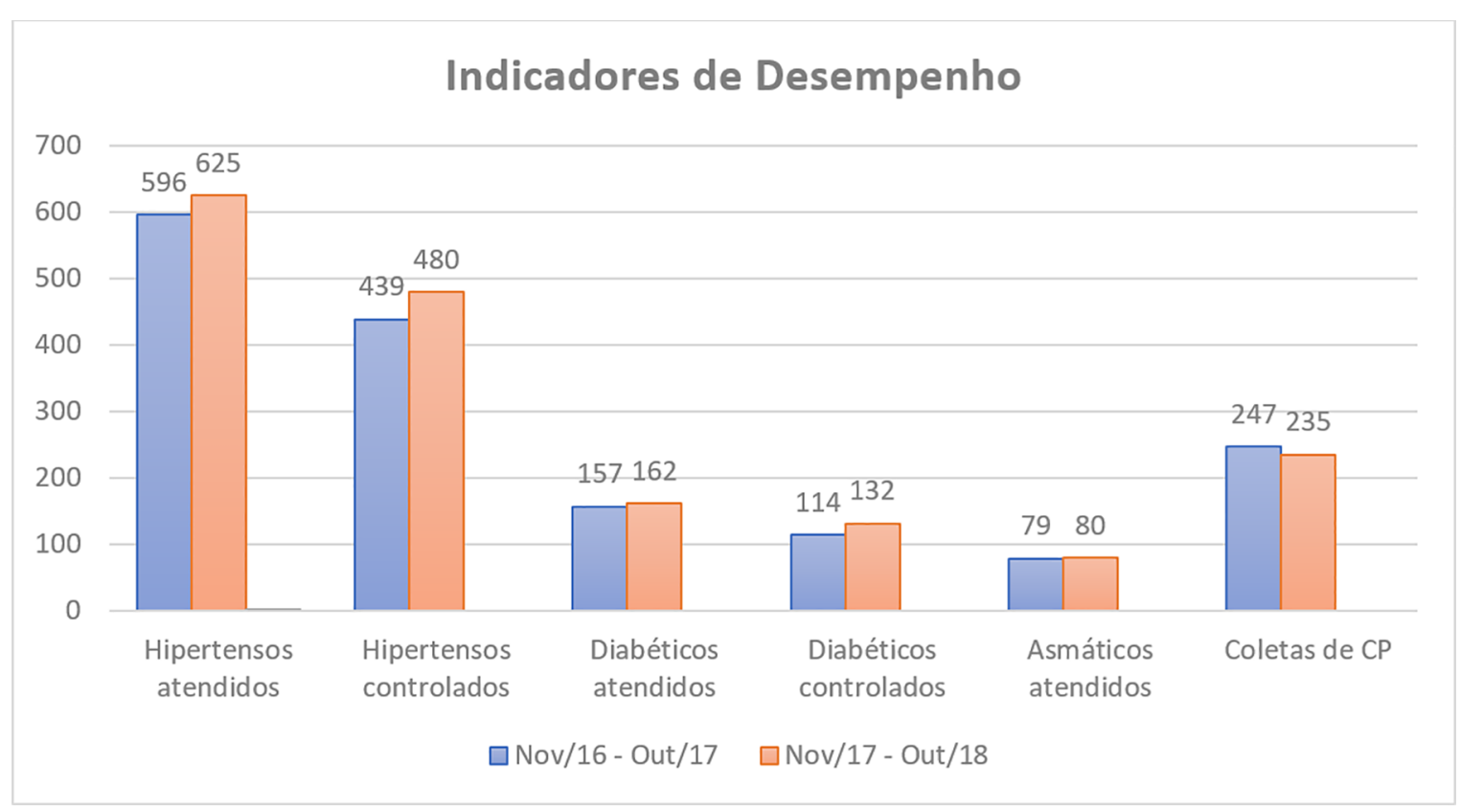

Gráfico 2. Indicadores de desempenho antes e após a implantação do AA.

Houve uma diminuição de quase $10 \%$ de atendimentos a pacientes vinculados à unidade de saúde nos serviços de atenção secundária de referência, porém o número de internações por condições sensíveis à APS teve um leve aumento (Gráfico 3).

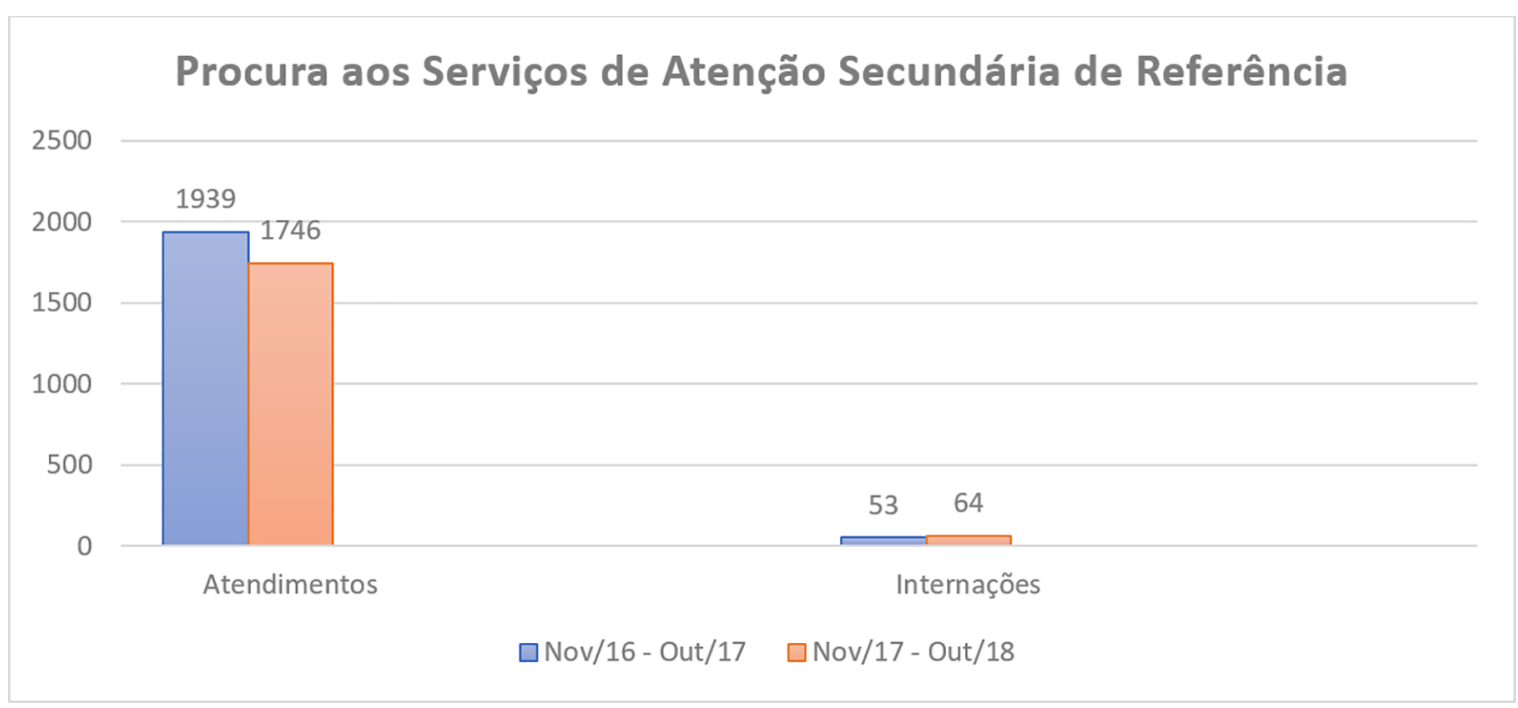

Gráfico 3. Procura aos serviços de atenção $2^{\underline{a}}$ de referência antes e após a implantação do AA.

\section{DISCUSSÃO}

A implantação do AA proporcionou um aumento no número de consultas médicas, como visto nos primeiros estudos internacionais ${ }^{8,9}$ e nacionais, ${ }^{10,11}$ além de ter diminuído a taxa de absenteísmo nas consultas, como descrito no estudo clínico randomizado de Belardi et al. ${ }^{12}$ e no estudo transversal de Asfor et al. ${ }^{13}$. 
Além disso, houve um crescimento no número de pacientes diferentes atendidos, o que fortalece a ideia de que esse modelo ampliou o acesso da população aos cuidados de saúde e não apenas concentrou mais atendimentos, para os mesmos pacientes, como sugerido no estudo de Vidal ${ }^{14}$.

Em relação aos cuidados de doenças crônicas, houve um leve aumento no número de diabéticos e hipertensos controlados, corroborando resultados vistos nos estudos de Sperl-Hillen et al. ${ }^{15}$ e Radel et al. ${ }^{16}$. O único programa que teve uma leve piora, com um decréscimo de cerca de $5 \%$ após a implantação do AA na unidade de saúde, foi a coleta de CP em mulheres entre 25-64 anos, fato que pode ter sido influenciado por alguns outros motivos como: mais mulheres fazendo este exame em outros serviços (hospitais ou clínicas particulares), diminuição da busca ativa das mulheres com exame atrasado e falta de proatividade por parte dos profissionais na oferta do exame.

Já em relação à procura aos serviços de atenção secundária, houve uma diminuição no número de atendimentos nos serviços de referência, como demonstrado no estudo de Solberg et al. ${ }^{17}$, apesar de ter ocorrido um leve aumento no número de internações após a implantação do AA. É importante destacar que não foi possível mensurar a quantidade de pacientes que procuraram outros serviços hospitalares e de emergência não vinculados ao GHC.

Como limitações deste estudo, podemos citar a sua metodologia transversal, que não permite inferências de causalidade entre o AA e os indicadores de desempenho. Além disso, o intervalo de um ano pode ser considerado um tempo pequeno para analisar e comparar os indicadores de acesso e desempenho da unidade, o ideal seria analisar por um período de tempo mais longo para se ter uma maior relevância dos resultados obtidos e diminuir um possível viés de sazonalidade.

Na literatura ainda não existem muitos estudos sobre o AA na atenção primária, em uma revisão sistemática ${ }^{18}$ de 2011, foram encontrados apenas vinte e quatro estudos quantitativos, a maioria dos EUA. No Brasil, foram encontrados apenas cinco estudos ${ }^{10,11,13,14,19}$ até este ano, o que fortalece a importância de que sejam feitas mais pesquisas sobre este tema.

Por fim, é possível considerar que a implantação do AA na US Sesc foi associada a uma melhoria dos indicadores de acesso, bem como da maior parte dos indicadores de desempenho analisados. Assim, sugere-se que sejam feitos esforços para que esse modelo de acesso possa ser implantado em outras unidades de saúde, principalmente as do SSC-GHC, e seu impacto seja continuamente monitorado através de outros estudos.

Colaboradores: Concepção e/ou delineamento do estudo: PPGB e LW. Aquisição, análise ou interpretação dos dados: PPGB e LW. Redação preliminar: PPGB. Revisão crítica da versão preliminar: LW. Todos os autores aprovaram a versão final e concordaram com prestar contas sobre todos os aspectos do trabalho.

Este trabalho foi aprovado pelo Comitê de Ética e Pesquisa do Grupo Hospitalar Conceição (parecer $\left.n^{\circ} 3.281 .396\right)$ em 24/04/2019. 


\section{REFERÊNCIAS}

1. Starfield B. Acessibilidade e primeiro contato: “a porta”. In: Starfield B, org. Atenção primária: equilíbrio entre necessidades de saúde, serviços e tecnologia. Brasília (DF): UNESCO/Ministério da Saúde; 2002. p. 207-45.

2. Lippman H. Same-day scheduling. Hippocrates. 2000;2:49-53.

3. Murray M, Berwick DM. Advanced access: reducing waiting and delays in primary care. JAMA. 2003;289:1035-40. PMID: 12597760 DOI: https://doi.org/10.1001/jama.289.8.1035

4. Mendes EV. O acesso à atenção primária em saúde. Brasília (DF): Conselho Nacional de Secretários de Saúde (CONASS); 2017.

5. Murray M, Tantau C. Same-day appointments: exploding the access paradigm. Fam Pract Manag. 2000 Set;7(8):45-50.

6. Knight AW, Padgett J, George B, Datoo MR. Reduced waiting times for the GP: two examples of advanced access in Australia. Med J Aust. 2005 Jul;183(2):101-3. PMID: 16022626 DOI: https://doi.org/10.5694/j.1326-5377.2005.tb06941.x

7. Witt MJ. Advanced access works! Improved patient satisfaction, access, and P4P scores. El Segundo, CA: Greenbranch Publishing; 2006.

8. Mallard SD, Leakeas T, Duncan WJ, Fleenor ME, Sinsky RJ. Same-day scheduling in a public health clinic: a pilot study. J Public Health Manag Pract. 2004 Mar/Apr;10(2):148-55. DOI: https://doi.org/10.1097/00124784-200403000-00009

9. Kennedy JG, Hsu JT. Implementation of an open access scheduling system in a residency training program. Fam Med.2003 Out;35(9):666-70.

10. Leal AEB, Watanabe BT, Bezerra CW, Palluello RAS, Gyuricza JV, Rewa T, et al. Acesso avançado: um caminho para a integridade na atenção básica. São Paulo (SP): Secretaria Municipal de Saúde/Coordenadoria Regional de Saúde Oeste; 2015.

11. Arrojo Junior JC, Fabi LF. Impacto do acesso avançado na capacidade de agendamento futuro em unidade básica de saúde do município de São Paulo. Med (Ribeirão Preto). 2014;47(Suppl 5):19.

12. Belardi FG, Weir S, Craig FW. A controlled trial of an advanced access appointment system in a residency family medicine center. Fam Med. 2004 Mai;36(5):341-5.

13. Asfor ATP, Shinkai MP, Monteiro ABC, Freitas HC, Shinkai H. Implantação do acesso avançado como medida resolutiva ao absenteísmo às consultas programadas. In: Anais do Congresso Sul-Brasileiro de Medicina de Família e Comunidade. 12th WONCA World Rural Health Conference; 3-5 abr 2014; Gramado, Rio Grande do Sul, Brasil. Gramado (RS): WONCA/SBMFC; 2014.

14. Vidal TB. O acesso avançado e sua relação com o número de atendimentos médicos em atenção primária à saúde [dissertação]. Porto Alegre (RS): Universidade Federal do Rio Grande do Sul (UFRGS) - Programa de Pós-Graduação em Epidemiologia; 2013.

15. Sperl-Hillen JM, Solberg LI, Hroscikoski MC, Crain AL, Engebretson KI, O'Connor PJ.The effect of advanced access implementation on quality of diabetes care. Prev Chronic Dis. 2008 Jan;5(1):A16.

16. Radel SJ, Norman AM, Notaro AM, Horrigan DR. Redesigning clinical office practices to improve performance levels in an individual practice association model HMO. J Healthc Qual. 2001 Mar/Apr;23(2):11-5. DOI: https://doi.org/10.1111/j.1945-1474.2001.tb00330.x

17. Solberg LI, Maciosek MV, Sperl-Hillen JM, Carin AL, Engebretson KI, Asplin BR, et al. Does improved access to care affect utilization and costs for patients with chronic conditions?. Am J Manag Care. 2004 Out;10(10):717-22.

18. Rose D, Ross JS, Horwitz LI. Advanced access scheduling outcomes: a systematic review. Arch Intern Med. 2011 Jul;171(13):1150-9. DOI: https://doi.org/10.1001/archinternmed.2011.168

19. Pires Filho LAS, Azevedo-Marques JM, Duarte NSM, Moscovici L. Acesso avançado em uma Unidade de Saúde da Família do interior do estado de São Paulo: um relato de experiência. Saúde Debate. 2019 Abr;43(121):605-13. DOI: https://doi.org/10.1590/01031104201912124 